\title{
VME Data Acquisition Modules for MINERvA Experiment
}

\author{
Boris Baldin \\ Fermi National Accelerator Laboratory, Batavia, IL 60510
}

\begin{abstract}
This document describes two VME modules developed for MINERvA experiment at Fermilab. The Chain ReadOut Controller (CROC) module has four serial data channels and can interface with up to 48 frontends using standard CAT5e networking cable. The data transmission rate of each channel is $160 \mathrm{Mbit} / \mathrm{s}$. The maximum data transmission rate via VME bus is $\sim 18 \mathrm{MB} / \mathrm{s}$. The Chain Readout Interface Module (CRIM) is designed to provide various interface functions for the CROC module. It is compatible with MINOS MTM timing module and can be used to distribute timing signals to four CROC modules. The CRIM module also has a data port compatible with the CROC serial data interface. The data port can be used for diagnostic purpose and can generate triggers from front-end events. The CRIM module is a standard D08(O) interrupter module.
\end{abstract}




\section{MINERvA Chain Readout Controller}

\section{Introduction}

MINERvA Chain Readout Controller (CROC) is dedicated to provide timing, slow control and data readout for front-end modules based on 64-channel multi-anode photomultiplier tube. The front-end modules are connected to each other in a chain using commercial networking cable. Both ends of the chain or DAQ loop are connected to the Chain Readout Controller. The Chain Readout Controller is a VME based module which resides in a VME crate controlled by a PC via commercial PCI-VME interface or by an embedded VME controller. The Chain Readout Controller receives a set of MINOS timing signals from a modified version of the MINOS MTM module ${ }^{[1]}$.

\section{DAQ loop}

The DAQ loop consists of up to 12 front-ends. The number of front-ends in the loop and the total cable length are limited by the degradation of the timing signal which is common for all front-ends in the loop. The maximum length of the loop should not exceed $60 \mathrm{ft}$. A diagram of the DAQ loop signals is shown in Fig. 1.

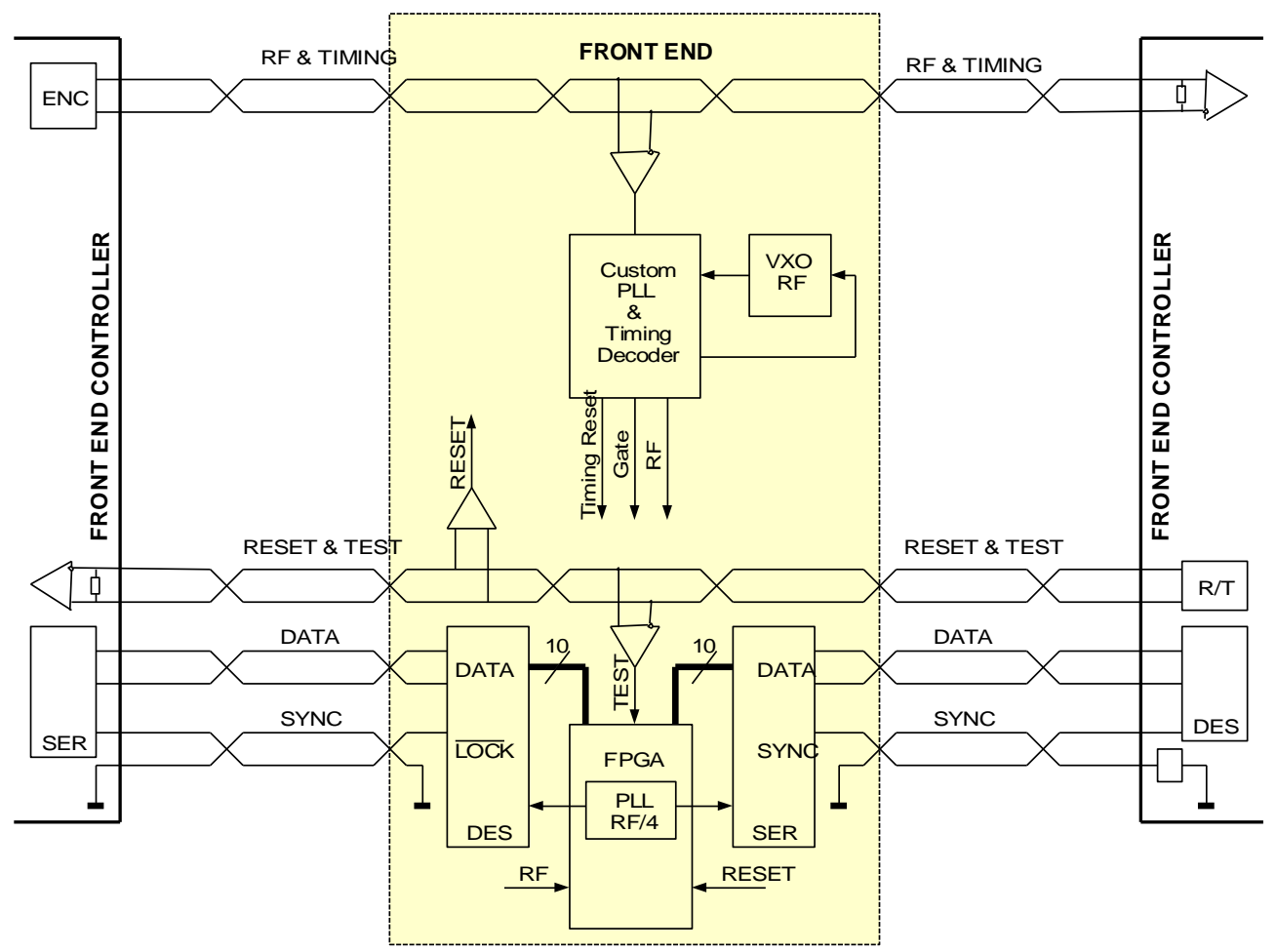

Fig. 1 Diagram of the DAQ loop signals

There are four signals in the DAQ loop. Two of the signals "RF \& Timing" and "Reset \& Test" are bussed (multi-drop) signals that propagate through the front-ends via continuous 
twisted pair. There is one driver and one receiver at the controller side and multiple receivers in the front-ends for these signals. The other two signals "Data" and "Sync" are chained (point-to-point) signals that are received and transmitted by each front-end. "RF \& Timing" and "Data" propagate in one direction, and "Reset \& Test" and "Sync" propagate in the opposite direction.

The "RF \& Timing" signal is an accelerator RF clock signal with embedded encoded timing signals. This signal is generated by a standard LVDS driver and passes through all front-ends in a loop until it reaches an LVDS receiver at the controller. The implementation of the timing encoding is similar to the D0 muon timing encoding scheme developed by Sten Hansen ${ }^{[2]}$. A timing diagram of the encoded signals is shown in Fig. 2. The FCMND signal is an example of a software generated timing command.

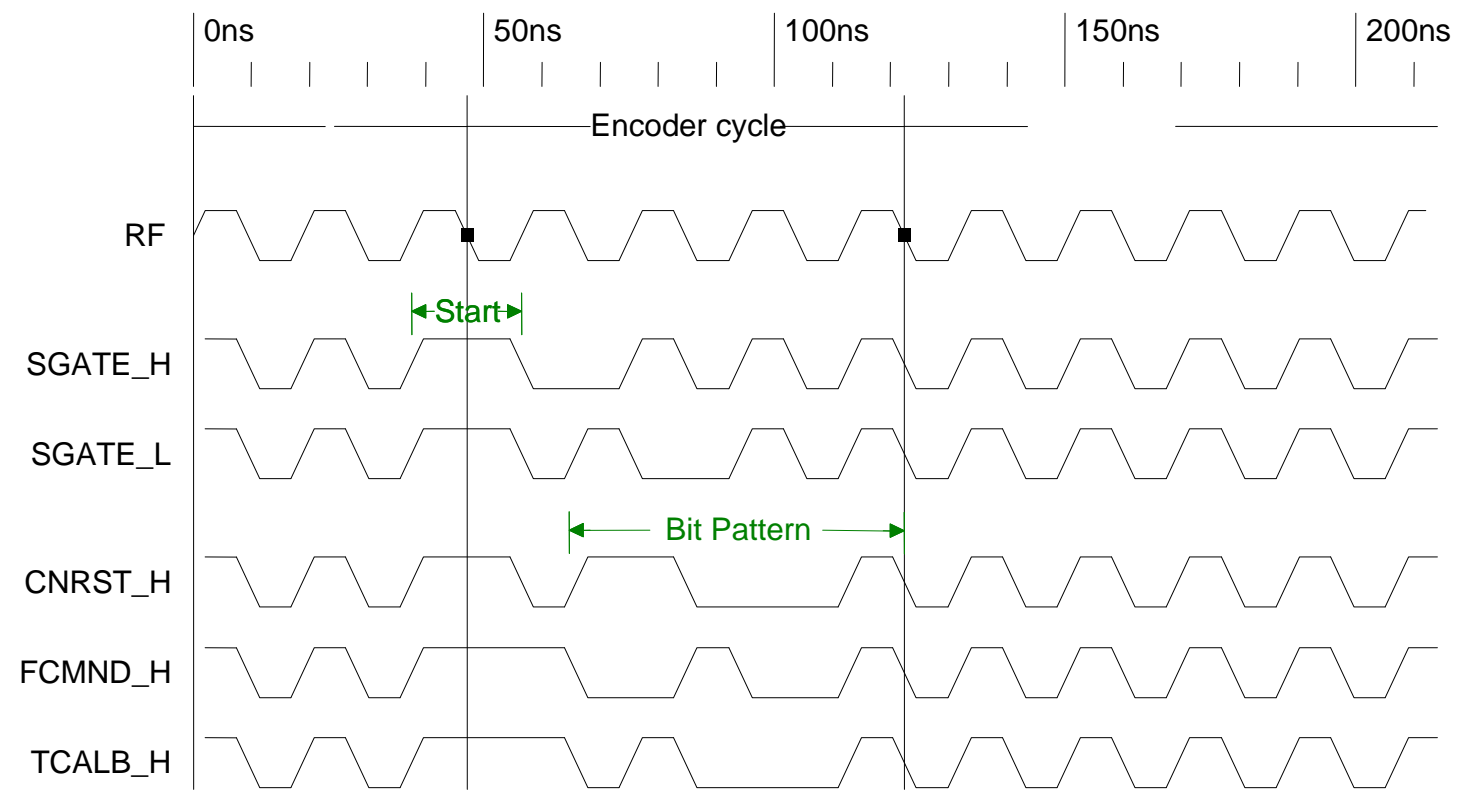

Fig. 2 Timing Encoder signals

The encoded timing signal always starts with two consecutive ones and ends with a one. A seven bit pattern follows the start bits. Note that not all bit combinations are acceptable. Table 1 shows actual binary vales of the encoded MTM signals.

Table 1 Encoded timing signals

\begin{tabular}{|l|l|l|l|l|}
\hline No. & Signal Name & Binary Value & Hex Value & Comment \\
\hline 1 & SGATE_H & 10110001 & $0 \times B 1$ & Rising edge \\
\hline 2 & SGATE_L & 11010001 & $0 x D 1$ & Falling edge \\
\hline 3 & CNRST_H & 11000101 & $0 \times C 5$ & Rising edge \\
\hline 4 & TCALB_H & 10001001 & $0 \times 89$ & Rising edge \\
\hline
\end{tabular}


The proposed scheme allows encoding up to 34 different signals and has a fixed delay of 5.5 RF clock cycles. Additional timing signals can be generated by issuing a VME write command (Fast command) to an internal register of the module.

The duration of the "Reset \& Test" LVDS signal is different for these two signals. The test pulse duration is $\sim 20 \mathrm{nS}$ and the reset pulse duration is $\sim 100 \mu \mathrm{S}$. The front-ends detect the reset pulse and perform cold start reset. The test signal is used to measure propagation delay of the cable loop and for testing front-end's functionality. It can be applied at the logic level or used to fire an analog test pulser located at the front-end board. The reset signal is used explicitly for resetting front-ends to a default state.

The "Data" signal is an LVDS output of the Texas Instruments SN65LV1023A serializer chip. A matching SN65LV1224B deserializer chip is used in the front-end circuitry and receiving part of the controller. The clock frequency selected for data transmission is $\mathrm{RF} / 4$ or $13.28 \mathrm{MHz}$ which lowers the power consumption of both chips to a reasonable level $(<60 \mathrm{~mW})$ and sets the bit frequency at $159.31 \mathrm{MHz}$ which allows to extend the length of the cable loop without a risk of degrading the performance.

Table 2 DAQ loop signal specification

\begin{tabular}{|l|l|l|l|l|l|}
\hline No. & Signal & Level & Direction & Termination & Comment \\
\hline 1 & RF \& Timing & LVDS & Downstream & Controller & Bussed \\
\hline 2 & Reset \& Test & LVDS & Upstream & Controller & Bussed \\
\hline 3 & Data & LVDS & Downstream & Front-End & Chained \\
\hline 4 & Sync & LVTTL & Upstream & N/A & GND \\
\hline
\end{tabular}

Table 3. Serializer port pinouts

\begin{tabular}{|l|l|l|l|}
\hline No. & Signal Name & Pin & Comment \\
\hline 1 & SRFTM+ & 1 & LVDS output \\
\hline 2 & SRFTM- & 2 & LVDS output \\
\hline 3 & SRTST+ & 4 & LVDS input, terminated \\
\hline 4 & SRTST- & 5 & LVDS input, terminated \\
\hline 5 & DOUT+ & 7 & LVDS output \\
\hline 6 & DOUT- & 8 & LVDS output \\
\hline 7 & SYNC & 3 & LVTTL input, no termination \\
\hline 8 & GND & 6 & Connected to GND via 100 ohms \\
\hline
\end{tabular}

Note: RFTIM+, RFTIM-, RSTST+ and RSTST- are connected to same name pins on the front-end's connectors. 
The "Sync" signal is a deserializer LVTTL "Lock" output of the PLL. This connection allows for automatic re-synchronization of the deserializer. If the lock is lost, serializer will generate synchronization pattern until deserializer is locked again. The controller and front-ends have status bits of the connection upstream and downstream. Summary of four DAQ loop signals is presented in Table 2 and pinouts of the dual port RJ-45 jack in Table 3 and Table 4.

Table 4 Deserializer port pinouts

\begin{tabular}{|l|l|l|l|}
\hline No. & Signal Name & Pin & Comment \\
\hline 1 & DRFTM+ & 1 & LVDS input, terminated \\
\hline 2 & DRFTM- & 2 & LVDS input, terminated \\
\hline 3 & DRTST+ & 4 & LVDS output \\
\hline 4 & DRTST- & 5 & LVDS output \\
\hline 5 & DATIN+ & 7 & LVDS input, terminated \\
\hline 6 & DATIN- & 8 & LVDS input, terminated \\
\hline 7 & LOCKB & 3 & LVTTL output active low \\
\hline 8 & GND & 6 & Connected to GND via 100 ohms \\
\hline
\end{tabular}

The Chain Readout Controller receives timing signals from the MINOS Master Clock System. A modified version of the MINOS Minder Timing Module (MTM) is used to distribute timing signals within MINERvA readout crates. Each CROC has an input CAT5e RJ-45 connector compatible with a set of standard MINOS timing signals. The pinouts of the connector are shown in Table 5.

Table 5 Timing input connector pinouts

\begin{tabular}{|l|l|l|l|}
\hline No. & Signal Name & Pin & Comment \\
\hline 1 & RF- & 1 & LVDS input, terminated \\
\hline 2 & RF+ & 2 & LVDS input, terminated \\
\hline 3 & SGATE- & 3 & LVDS input, terminated \\
\hline 4 & SGATE+ & 4 & LVDS input, terminated \\
\hline 5 & CNRST- & 5 & LVDS input, terminated \\
\hline 6 & CNRST+ & 6 & LVDS input, terminated \\
\hline 7 & TCALB- & 7 & LVDS input, terminated \\
\hline 8 & TCALB+ & 8 & LVDS input, terminated \\
\hline
\end{tabular}

Note: The pinouts of the connector correspond to CAT3 straight pinouts 
A simplified block-diagram of the data transmission logic is shown in Fig. 3. The contents of the message first must be loaded into a 16-bit wide FIFO memory via VME bus. After the message is loaded, it can be transmitted by sending a VME command to the controller. After the front-end has responded, its message can be read out from a Dual Port Memory (DPM). There is a status register for each DAQ loop channel with various status information including FIFO Empty flag, FIFO Full flag, Message Sent, Message Received bits etc.

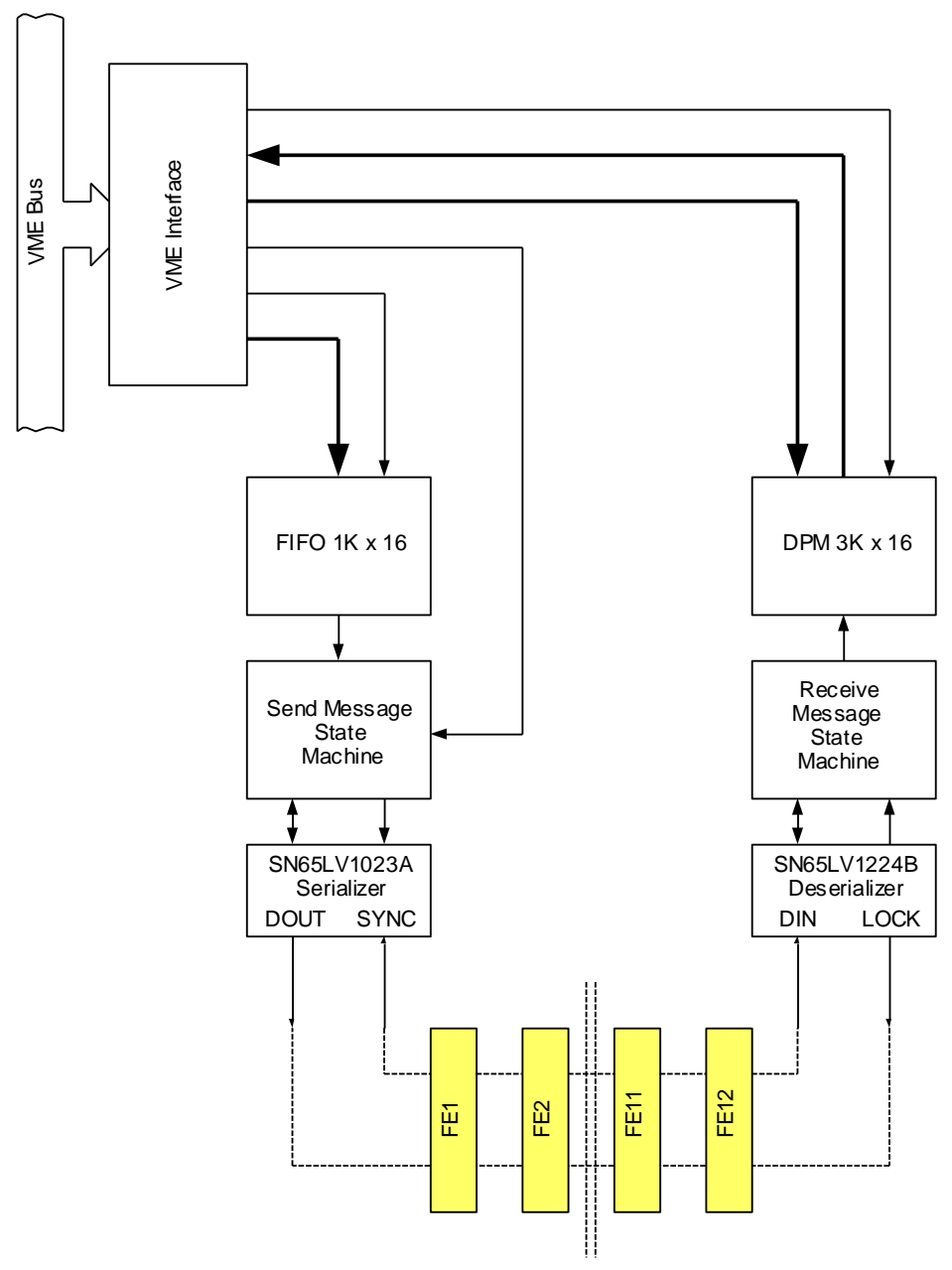

Fig. 3 Data transmission logic

\section{Data formats}

A data from the controller is transferred to front-ends in a form of messages. Each message consists of several groups of 10-bit words ${ }^{[3]}$. Two upper bits of the word are control bits used to indicate the beginning and the end of a message and lower eight bits represent actual byte of data. Currently there are four groups in a message. First group consists of one word. This group also indicates the beginning of a message and includes an address of the front-end. Second group consists of eight words. Third group is actual data send to the front-end. The number of words in this group is always odd. The 
readout controller also attaches a CRC byte as a fourth group at the end of the message for data verification. This group also indicates the end of a message. The total number of bytes that controller receives from the VME master as a message data is always even. The order of bytes in a 16-bit word follows Big Endian VME byte ordering. In the DAQ loop the most significant byte is transmitted first.

Each front-end in the loop has a unique address. The front-end re-transmits the message if the address in the message does not match its pre-assigned address. The addressed front-end responds to a controller's message by transmitting its response along the chain back to the controller. The format of the response message follows the same rules as described in the previous paragraph. There is always odd number of words in the third group, so the total number of data bytes received by the controller excluding CRC byte is always even. This convention simplifies formatting data to 16-bit VME words. The controller calculates the number of data bytes it receives and writes it at a starting address of the DPM as a 16-bit data word. The length of the data in bytes includes itself. Consequent messages are written starting at the current value of the address pointer with the data length first. The overflow bit is set when the address pointer reaches the last available address. The DPM memory is accessible to the VME master in 16-bit and 32bit data formats. The received CRC byte is compared to the calculated checksum of the data, but the DPM address pointer is not incremented. Several messages can be stored in the DPM for the consequent readout. The size of the DPM for each DAQ loop is $6 \mathrm{~KB}$. The size of the FIFO memory for each channel is $2 \mathrm{~KB}$.

\section{VME module implementation}

The current version of the readout controller is implemented as a

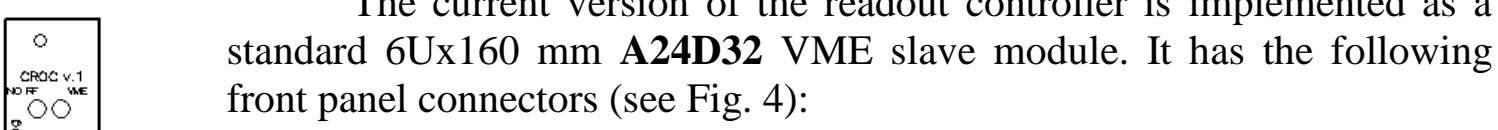

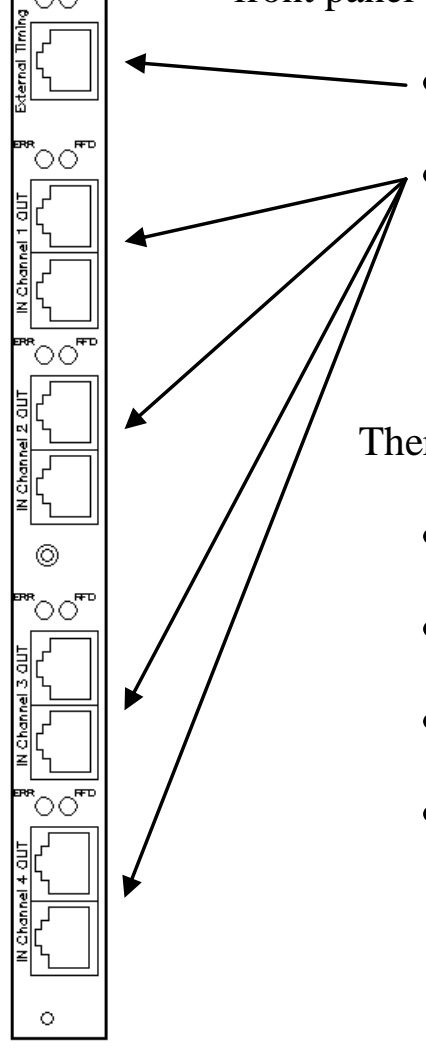

A single RJ-45 input timing connector

- Four dual RJ-45 DAQ loop connectors. Note that the top connector of the pair has to be connected to the first frontend in a chain

Fig. 4 CROC front panel

- "VME" (green) - VME command decoded

- "NO RF" (red) - missing external RF input signal

- "RFD” (green) - RF signal detected in the loop

- "ERR" (red) - CE, TO or DF error bit is set 


\section{Specification}

- Power consumption

- External timing inputs

- DAQ loop signals

- Main clock frequency

- VME address modifiers

- VME address/data mode

- DPM read address/data mode

- Number of front-ends per loop

- Channel FIFO size

- Channel DPM size

- DAQ loop cable type

- Maximum loop length

- DAQ loop data rate
$-+5 \mathrm{~V} / 1.2 \mathrm{~A}$

- LVDS (see Table 5)

- LVDS/LVTTL (see Table 2)

- $53.1 \mathrm{MHz}( \pm 200 \mathrm{ppm})$

- 39, 3d, 3e, 3a, 3b, 3f

- A24D16

- A24D16, A24D32, A24BLT

-12 max

- $1 \mathrm{Kx} 16$ bit

- 3Kx16 bit

- unshielded CAT5e

- $60 \mathrm{ft}$.

- 160 Mbit/s (12 bits @13.3 MHz)

\section{VME registers}

Each controller module has four DAQ loops or channels. Each channel has its own unique VME addresses for memories and data registers. An eight bit on-board switch selects $64 \mathrm{~K}$ address space for each controller. A general VME memory map is shown in Table 7. The description of the readout controller channel data registers listed below is the same for all channels $(n=1-4)$.

Readout Controller channel input data register MDatan

\begin{tabular}{|l|l|l|l|l|l|l|l|l|l|l|l|l|l|l|l|}
\hline \multicolumn{10}{|c|}{ VME Data bits } \\
\hline 15 & 14 & 13 & 12 & 11 & 10 & 9 & 8 & 7 & 6 & 5 & 4 & 3 & 2 & 1 & 0 \\
\hline \multicolumn{10}{|c|}{ BYTE(0) } & \multicolumn{10}{|c|}{} \\
\hline
\end{tabular}

Note: BYTE $(0)$ - first byte of the message, BYTE $(1)$ - second byte of the message.

In order to send a message to the front-end, a VME master has to write all data bytes to a CROC's FIFO memory using a single 16-bit data register. After all data is written, the Send Message command has to be issued by writing 0x0101 to the Send Message register. The controller will wait for the front-end response and indicate that it has received it by changing the Message Received status bit. If the CRC sum was incorrect, the CRC error bit will be set as well. If the controller does not receive a response from a front-end within $\sim 480 \mu \mathrm{S}$, the TimeOut error bit is set. If the address pointer reaches $17 F F$, the DPM Full error bit is set.

Readout Controller channel Send Message register SM $n$

\begin{tabular}{|l|l|l|l|l|l|l|l|l|l|l|l|l|l|l|l|}
\hline \multicolumn{11}{|c|}{ VME Data bits } \\
\hline 15 & 14 & 13 & 12 & 11 & 10 & 9 & 8 & 7 & 6 & 5 & 4 & 3 & 2 & 1 & 0 \\
\hline 0 & 0 & 0 & 0 & 0 & 0 & 0 & 1 & 0 & 0 & 0 & 0 & 0 & 0 & 0 & 1 \\
\hline
\end{tabular}


The output data format is similar to the input data format. First word of the data is data length in bytes (inclusive). The most significant byte of the message is received first.

Readout Controller channel received data Dual Port Memory RDatan

\begin{tabular}{|c|c|c|c|c|c|c|c|c|c|c|c|c|c|c|c|}
\hline \multicolumn{16}{|c|}{ VME Data bits } \\
\hline 15 & 14 & 13 & 12 & 11 & 10 & 9 & 8 & 7 & 6 & 5 & 4 & 3 & 2 & 1 & 0 \\
\hline \multicolumn{8}{|c|}{$\mathrm{DL}(0)$} & \multicolumn{8}{|c|}{$\mathrm{DL}(1)$} \\
\hline \multicolumn{8}{|c|}{ BYTE(0) } & \multicolumn{8}{|c|}{ BYTE(1) } \\
\hline \multicolumn{8}{|c|}{$\ldots$} & \multicolumn{8}{|c|}{$\ldots$} \\
\hline \multicolumn{8}{|c|}{$\operatorname{BYTE}(2 \mathrm{k}-1)$} & \multicolumn{8}{|c|}{$\operatorname{BYTE}(2 \mathrm{k})$} \\
\hline
\end{tabular}

Note: DL1, DL0 - data length, BYTE(0) - first byte of the message, BYTE(1) second byte of the message, BYTE(2k) - last (even) byte of the message.

The received data can be read out from the controller Dual Port Memory using specified address range for each channel.

Readout Controller channel Status Register SR $n$

\begin{tabular}{|l|l|l|l|l|l|l|l|l|l|l|l|l|l|l|l|}
\hline \multicolumn{11}{|c|}{ VME Data bits } \\
\hline 15 & 14 & 13 & 12 & 11 & 10 & 9 & 8 & 7 & 6 & 5 & 4 & 3 & 2 & 1 & 0 \\
\hline 0 & 0 & PL1 & PLO & 0 & LS & SY & RF & 0 & DF & FF & EF & TO & CE & MR & MS \\
\hline
\end{tabular}

Note: MS - Message sent,

MR - Message received,

CE - CRC error,

TO - Timeout error,

EF $\quad-$ FIFO not empty flag (0 - empty),

FF $\quad-$ FIFO full flag $(1-$ full $)$,

DF $\quad-$ DPM full flag $(1-$ full $)$,

RF $\quad-$ RF loop monitor (1 - RF present),

SY - Serializer SYNC status (1 - synchronized),

LS - Deserializer LOCK status (1 - locked),

PL0 - CPLD PLL0 status (1 - locked)

PL1 - CPLD PLL1 status (1 - locked).

A separate VME command is used to clear some status register bits. Only seven bits: MS, MR, CE, TO, FF, EF and DF are cleared by this command. The DPM write address pointer has to be reset after each front-end message is read out, otherwise the next message will be stored at the next available address at the end of the previous message. Both commands are using the same VME address, but different data bits as shown below. 
Readout Controller channel Clear Status/Reset DPM register CSn

\begin{tabular}{|l|l|l|l|l|l|l|l|l|l|l|l|l|l|l|l|}
\hline \multicolumn{11}{|c|}{ VME Data bits } \\
\hline 15 & 14 & 13 & 12 & 11 & 10 & 9 & 8 & 7 & 6 & 5 & 4 & 3 & 2 & 1 & 0 \\
\hline 0 & 0 & 0 & 0 & RP & 0 & CS & 0 & 0 & 0 & 0 & 0 & RP & 0 & CS & 0 \\
\hline
\end{tabular}

Note: $\mathrm{CS}=1$ for Clear Status command, otherwise 0

$\mathrm{RP}=1$ for Reset DPM pointer command, otherwise 0

In order to facilitate cable loop delay measurement, which is necessary for a correction of the SGATE propagation delay to the front-end the test pulse is used. The loop delay counter is running at $8 \mathrm{RF}$ frequency, which provides a timing resolution of $\sim 2.35 \mathrm{nS}$. Each time the test pulse is generated by the CROC logic, the counter is loaded with the time difference between its departure and arrival. The cable delay value is stored until Clear Status command is issued. It is possible to perform multiple measurements by issuing test pulse several times and dividing the result by the number of pulses. The result of this measurement combined with the measured by the front-end arrival time of the test pulse can be used to calculate individual propagation delay of the timing signals to each front-end in the DAQ loop. Assuming that the front-end is capable of measuring a time difference between rising edge of the SGATE signal and arrival time of the test pulse, the following equation is true:

$$
T_{f}=\left(T 0+T_{d}+T_{1}-\mathbf{M 0}\right) / 2 \text {, where }
$$

$\mathbf{T}_{\mathbf{f}}$ - propagation delay of SGATE to the front-end,

T0 - delay setting of the test pulse relative to SGATE,

$\mathbf{T}_{\mathbf{d}} \quad$ - internal logic delay (will be measured with the front-end),

$\mathbf{T}_{\mathbf{l}} \quad$ - cable loop delay,

M0 - test pulse arrival time relative to SGATE.

Readout Controller channel Loop Delay register LD $n$

\begin{tabular}{|l|l|l|l|l|l|l|l|l|l|l|l|l|l|l|l|}
\hline \multicolumn{11}{|c|}{ VME Data bits } \\
\hline 15 & 14 & 13 & 12 & 11 & 10 & 9 & 8 & 7 & 6 & 5 & 4 & 3 & 2 & 1 & 0 \\
\hline 0 & D6 & D5 & D4 & D3 & D2 & D1 & D0 & 0 & 0 & 0 & 0 & 0 & 0 & 0 & 0 \\
\hline
\end{tabular}

Note: D6..D0 - result of the loop delay measurement in $2.35 \mathrm{nS}$ steps

It is possible to accumulate several messages in the DPM, assuming they will require less than $6 \mathrm{~K}$ bytes of storage. In order to read the whole array of messages in a single step one need to know what is the size of the array. There is a separate VME command to read current value of the DPM write pointer. The actual size of the array is equal to the value of the DPM pointer minus two bytes. 
Readout Controller channel DPM pointer register MPn

\begin{tabular}{|l|l|l|l|l|l|l|l|l|l|l|l|l|l|l|l|}
\hline \multicolumn{11}{|c|}{ VME Data bits } \\
\hline 15 & 14 & 13 & 12 & 11 & 10 & 9 & 8 & 7 & 6 & 5 & 4 & 3 & 2 & 1 & 0 \\
\hline P7 & P6 & P5 & P4 & P3 & P2 & P1 & P0 & P15 & P14 & P13 & P12 & P11 & P10 & P9 & P8 \\
\hline
\end{tabular}

Note: P0..P15 - current value of the DPM write pointer (bytes)

The following control registers are common for all four channels. The Timing Setup register allows selecting internal or external clock source and time delay of the test pulse. In the internal mode main clock signal is generated using $53 \mathrm{MHz}$ quartz crystal. In the external mode the MINOS MTM module can be used as an external clock source. The test pulse is generated every time when positive transition of the SGATE signal is detected and the Test Pulse delay is enabled. A relative delay of the pulse can be selected using bits D0-D9 of the Timing Setup register. Writing to the Test Pulse register also generates a test pulse. Writing to the Channel Reset register generates a channel reset pulse. Both test pulse and channel reset pulse will be sent only to the channels with the corresponding mask bit set to one. Writing to the Fast Command register an 8-bit value generates an encoded timing command. The following additional timing commands are currently used in the front-end:

Table 6 Additional encoded timing commands

\begin{tabular}{|l|l|c|c|}
\hline No. & Signal Name & Binary Value & F7..F0 hex value \\
\hline 5 & FPGA_RST & 10001101 & 0x8D \\
\hline 6 & LOAD_TIMER & 11001001 & 0xC9 \\
\hline
\end{tabular}

The MTM signals listed in the Table 1 can be simulated using this command as well. One should keep in mind that in this case they will not be synchronous to the MINOS timing. Generating SGATE using software timing commands will not generate the delayed test pulse even if the Test Pulse delay is enabled. The Test Pulse delay enable bit can be used to disable the test pulse while running with the external SGATE signal.

Readout Controller Timing Setup register TS0

\begin{tabular}{|l|l|l|l|l|l|l|l|l|l|l|l|l|l|l|l|}
\hline \multicolumn{11}{|c|}{ VME Data bits } \\
\hline 15 & 14 & 13 & 12 & 11 & 10 & 9 & 8 & 7 & 6 & 5 & 4 & 3 & 2 & 1 & 0 \\
\hline CM & 0 & 0 & TE & 0 & 0 & D9 & D8 & D7 & D6 & D5 & D4 & D3 & D2 & D1 & D0 \\
\hline
\end{tabular}

Note: TE $\quad-$ Test Pulse delay enable $(1$ - enabled $)$

D0-D9 - Test Pulse delay value relative to SGATE in 18.9 ns steps

CM - Clock mode ( 1 - external, 0 - internal $)$ 
Readout Controller Reset and Test pulse mask register RT0

\begin{tabular}{|l|l|l|l|l|l|l|l|l|l|l|l|l|l|l|l|}
\hline \multicolumn{11}{|c|}{ VME Data bits } \\
\hline 15 & 14 & 13 & 12 & 11 & 10 & 9 & 8 & 7 & 6 & 5 & 4 & 3 & 2 & 1 & 0 \\
\hline 0 & 0 & 0 & 0 & R4 & R3 & R2 & R1 & 0 & 0 & 0 & 0 & T4 & T3 & T2 & T1 \\
\hline
\end{tabular}

Note: T1-T4 - Test pulse enable for channels 1-4 R1-R4 - Reset enable for channels 1-4

Readout Controller Channel Reset register CR0

\begin{tabular}{|l|l|l|l|l|l|l|l|l|l|l|l|l|l|l|l|}
\hline \multicolumn{11}{|c|}{ VME Data bits } \\
\hline 15 & 14 & 13 & 12 & 11 & 10 & 9 & 8 & 7 & 6 & 5 & 4 & 3 & 2 & 1 & 0 \\
\hline 0 & 0 & 0 & 0 & 0 & 0 & 1 & 0 & 0 & 0 & 0 & 0 & 0 & 0 & 1 & 0 \\
\hline
\end{tabular}

Readout Controller Fast Command register FC0

\begin{tabular}{|c|c|c|c|c|c|c|c|c|c|c|c|c|c|c|c|}
\hline \multicolumn{16}{|c|}{ VME Data bits } \\
\hline 15 & 14 & 13 & 12 & 11 & 10 & 9 & 8 & 7 & 6 & 5 & 4 & 3 & 2 & 1 & 0 \\
\hline$N C$ & NC & NC & NC & $N C$ & NC & NC & NC & F7 & F6 & F5 & F4 & F3 & F2 & F1 & FO \\
\hline
\end{tabular}

Readout Controller Test Pulse register TP0

\begin{tabular}{|l|l|l|l|l|l|l|l|l|l|l|l|l|l|l|l|}
\hline \multicolumn{11}{|c|}{ VME Data bits } \\
\hline 15 & 14 & 13 & 12 & 11 & 10 & 9 & 8 & 7 & 6 & 5 & 4 & 3 & 2 & 1 & 0 \\
\hline 0 & 0 & 0 & 0 & 0 & 1 & 0 & 0 & 0 & 0 & 0 & 0 & 0 & 1 & 0 & 0 \\
\hline
\end{tabular}

Table 7 Readout controller VME map

\begin{tabular}{|l|c|c|l|}
\hline \multicolumn{1}{|c|}{ Starting Address } & Size & \multicolumn{1}{c|}{ Read/Wri } & \multicolumn{1}{c|}{ Comment } \\
\hline Base + 000000 & $6 \mathrm{~K}$ & $\mathrm{R}$ & Channel 1 DPM memory, RData1 \\
\hline Base + 002000 & 2 & $\mathrm{~W}$ & Channel 1 FIFO input data register, MData1 \\
\hline Base + 002010 & 2 & $\mathrm{~W}$ & Channel 1 Send Message register, SM1 \\
\hline Base + 002020 & 2 & $\mathrm{R}$ & Channel 1 Status Register, SR1 \\
\hline Base + 002030 & 2 & $\mathrm{~W}$ & Channel 1 Clear Status/Reset DPM register, CS1 \\
\hline Base + 002040 & 2 & $\mathrm{R}$ & Channel 1 Loop Delay register, LD1 \\
\hline Base + 002050 & 2 & $\mathrm{R}$ & Channel 1 DPM Pointer register, MP1 \\
\hline
\end{tabular}




\begin{tabular}{|c|c|c|c|}
\hline Base +004000 & $6 \mathrm{~K}$ & $\mathrm{R}$ & Channel 2 DPM memory, RData2 \\
\hline Base +006000 & 2 & W & Channel 2 FIFO input data register, MData2 \\
\hline Base +006010 & 2 & W & Channel 2 Send Message register, SM2 \\
\hline Base +006020 & 2 & $\mathrm{R}$ & Channel 2 Status Register, SR2 \\
\hline Base +006030 & 2 & W & Channel 2 Clear Status/Reset DPM register, CS2 \\
\hline Base +006040 & 2 & $\mathrm{R}$ & Channel 2 Loop Delay register, LD2 \\
\hline Base +006050 & 2 & $\mathrm{R}$ & Channel 2 DPM Pointer register, MP2 \\
\hline Base +008000 & $6 \mathrm{~K}$ & $\mathrm{R}$ & Channel 3 DPM memory, RData3 \\
\hline Base + 00A000 & 2 & W & Channel 3 FIFO input data register, MData3 \\
\hline Base + 00A010 & 2 & W & Channel 3 Send Message register, SM3 \\
\hline Base + 00A020 & 2 & $\mathrm{R}$ & Channel 3 Status Register, SR3 \\
\hline Base + 00A030 & 2 & W & Channel 3 Clear Status/Reset DPM register, CS3 \\
\hline Base + 00A040 & 2 & $\mathrm{R}$ & Channel 3 Loop Delay register, LD3 \\
\hline Base + 00A050 & 2 & $\mathrm{R}$ & Channel 3 DPM Pointer register, MP3 \\
\hline Base $+00 C 000$ & $6 \mathrm{~K}$ & $\mathrm{R}$ & Channel 4 DPM memory, RData4 \\
\hline Base + 00E000 & 2 & W & Channel 4 FIFO input data register, MData4 \\
\hline Base + 00E010 & 2 & W & Channel 4 Send Message register, SM4 \\
\hline Base + 00E020 & 2 & $\mathrm{R}$ & Channel 4 Status Register, SR4 \\
\hline Base + 00E030 & 2 & W & Channel 4 Clear Status/Reset DPM register, CS4 \\
\hline Base + 00E040 & 2 & $\mathrm{R}$ & Channel 4 Loop Delay register, LD4 \\
\hline Base + 00E050 & 2 & $\mathrm{R}$ & Channel 4 DPM Pointer register, MP4 \\
\hline Base + 00F000 & 2 & $\mathrm{R} / \mathrm{W}$ & Timing Setup register, TSO \\
\hline Base + 00F010 & 2 & $\mathrm{R} / \mathrm{W}$ & Reset and Test pulse mask register, RTO \\
\hline Base + 00F020 & 2 & W & Channel Reset register, CRO \\
\hline Base + 00F030 & 2 & W & Fast Command register, FCO \\
\hline Base + 00F040 & 2 & W & Test Pulse register, TPO \\
\hline
\end{tabular}




\section{MINERvA CROC Interface Module}

\section{Introduction}

MINERvA Chain Readout Interface Module (CRIM) is designed to provide various interface functions for the CROC (Chain Readout Controller ${ }^{[4]}$ ) VME module used in the DAQ system and various test stand setups. The need for this module is dictated by a wide variety of applications the CROC module must be used for. Implementation of a full set of requirements in the CROC design would unavoidably complicate it and increase its cost as well as potentially decrease the ease of its use and the reliability. On the other hand, additional features needed for the test stand configurations are mostly useless in a standard DAQ configuration and, therefore, present additional overhead for the CROC design.

\section{Main features}

The CRIM has the following front panel features (Fig. 8):

- External timing input RJ-45 connector compatible with MINOS MTM ${ }^{[2]}$ output

- Two dual port timing output RJ-45 connectors compatible with the CROC external timing input

- Four dual LEMO connectors for four LVTTL input and four LVTTL output signals ( $1 \mathrm{~K}$ or $50 \mathrm{ohm}$ input termination, $1 \mathrm{~K}$ output load)

- One dual port RJ-45 test connector compatible with the CROC DAQ loop signals

- LED indicators for External RF, VME cycle, LVTTL inputs, LVTTL outputs and DAQ loop signals

The following timing modes of operation are implemented:

- MTM timing mode - timing signals are received from the MTM module and some are distributed via four RJ-45 ports to CROC modules

- External timing mode - external timing signals SGATE, CNRST and TCALB, applied to the LVTTL inputs, are synchronized to the internal $53.1047 \mathrm{MHz}$ quartz oscillator and distributed via four RJ-45 ports

- Internal timing mode - all output timing signals are generated by an internal sequencer in sync with a free running on-board 53.1047 MHz quartz oscillator

- External DAQ loop timing mode - the on-board quartz oscillator is locked to the DAQ loop RF clock with encoded timing signals

The following functionality is implemented in the on-board CPLD firmware:

- VME A24D16 slave interface

- VME D08(O) programmable interrupter

- PLL circuit to control a 53.1 MHz VCOX 
- Timing sequencer triggerable by MTM or LVTTL input signals

Using functionality described above, the following configurations are possible:

- LED test stand with external LED pulser synchronized to the event data readout by the CROC either using CRIM LVTTL trigger output signal to fire the pulser or using pulser logic level output connected to the CRIM LVTTL input to start readout

- Small data acquisition system running in external or internal timing mode with up to four CROC modules

- Full data acquisition system with MINERvA MTM module and Chain Readout Interface Module to generate VME interrupts using MTM signals

- A self-triggered DAQ system using CRIM trigger capability to generate VME interrupts using a trigger word received from a Front-End

- CROC test setup simulating front-end response using DAQ loop test connector

In all described above configurations synchronization of the data readout to detector events can be done either by using VME interrupts or by polling a VME register.

\section{CRIM Functional blocks}

The CRIM has several independent functional blocks along with a common VME A24D16 slave interface:

- $\mathrm{D} 08(\mathrm{O})$ interrupter module

- Timing module

- DAQ loop test module

The following sections describe each module separately.

- Interrupter module

The interrupter module has eight inputs assigned in the following order:

1. Input 0 - External trigger from input connector " $\mathrm{T}$ "

2. Input 1 - Rising edge of SGATE signal

3. Input 2 - Falling edge of SGATE signal

4. Input 3 - CNRST signal from any source

5. Input 4 - TCALB signal from any source

6. Input 5 - Front-End trigger word received

7. Input 6 - Reserved for future expansion 


\section{Input 7 - Reserved for future expansion}

Input 0 has the highest priority and Input 7 has the lowest. If there are two or more interrupts pending, they are processed in the order of priority. In order to work with VME interrupts, the VME master has to have an interrupt handler.

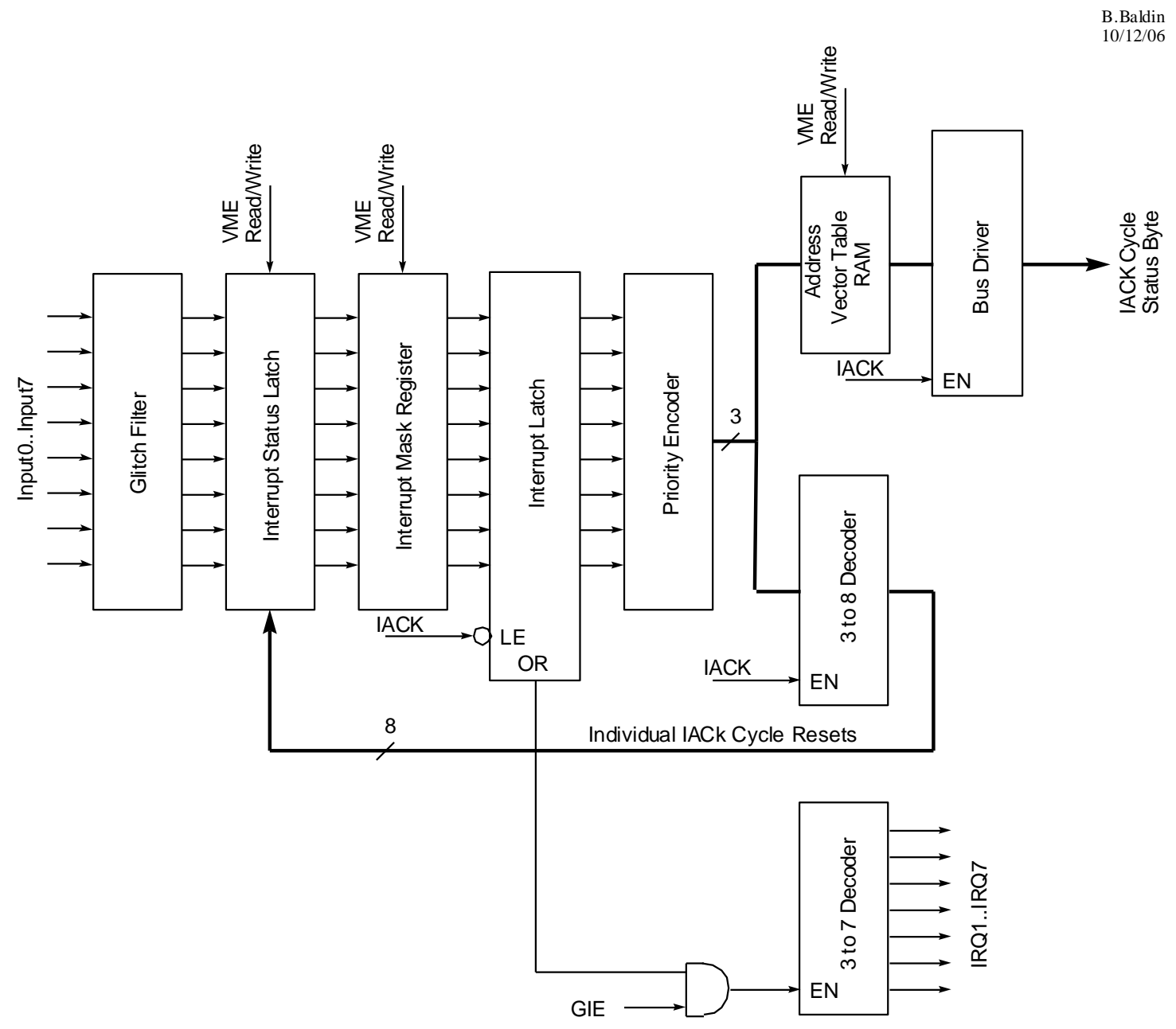

Fig. 5 Block Diagram of the CRIM Interrupter Logic

A block diagram of the CRIM Interrupter Logic is shown in Fig. 5. On power up, interrupts are disabled, interrupt level is set to five and interrupt vector table is loaded with default values (see Table 8 below). When GIE bit is set to one, interrupts are enabled. If any of the interrupt mask register bits is set to one, the corresponding interrupt input is enabled. When input signal sets an interrupt status bit to one, the IRQ line corresponding to the selected interrupt level is driven to zero. If corresponding software driver is active, an interrupt acknowledge cycle (IACK cycle) is triggered. During IACK cycle the interrupter logic compares three bit vector on address lines set by the interrupt handler with its programmed interrupt level. If there is a match, the interrupter puts eight bit status byte (status ID) on the data bus and clears corresponding interrupt status latch. 
After that the GIE bit is set to zero and has to be re-enabled by the software. All interrupter features are programmable via VME bus using the following registers:

Interrupt mask register IM, 0xF000

\begin{tabular}{|l|l|l|l|l|l|l|l|l|l|l|l|l|l|l|l|}
\hline \multicolumn{11}{|c|}{ VME Data bits } \\
\hline 15 & 14 & 13 & 12 & 11 & 10 & 9 & 8 & 7 & 6 & 5 & 4 & 3 & 2 & 1 & 0 \\
\hline 0 & 0 & 0 & 0 & 0 & 0 & 0 & 0 & M7 & M6 & M5 & M4 & M3 & M2 & M1 & M0 \\
\hline
\end{tabular}

Note: M0...M7 are individual mask bits corresponding Input $0 . . .7$ signals ( 1 - enable)

Interrupt status register IS, 0xF010

\begin{tabular}{|l|l|l|l|l|l|l|l|l|l|l|l|l|l|l|l|}
\hline \multicolumn{11}{|c|}{ VME Data bits } \\
\hline 15 & 14 & 13 & 12 & 11 & 10 & 9 & 8 & 7 & 6 & 5 & 4 & 3 & 2 & 1 & 0 \\
\hline 0 & 0 & 0 & 0 & 0 & 0 & 0 & 0 & 17 & 16 & 15 & 14 & 13 & 12 & 11 & 10 \\
\hline
\end{tabular}

Note: I0...I7 are pending interrupts corresponding Input $0 \ldots .7$ signals ( 1 - active), writing a one to any of I0...I7 bits will clear corresponding pending interrupt

Clear pending interrupts register $\mathrm{CP}, 0 \mathrm{xF} 020$

\begin{tabular}{|l|l|l|l|l|l|l|l|l|l|l|l|l|l|l|l|}
\hline \multicolumn{11}{|c|}{ VME Data bits } \\
\hline 15 & 14 & 13 & 12 & 11 & 10 & 9 & 8 & 7 & 6 & 5 & 4 & 3 & 2 & 1 & 0 \\
\hline 0 & 0 & 0 & 0 & 0 & 0 & 0 & 0 & 1 & 0 & 0 & 0 & 0 & 0 & 0 & 1 \\
\hline
\end{tabular}

Note: Writing 0x81 to this register will clear all pending interrupts

Interrupt configuration register $\mathrm{IC}, 0 \mathrm{xF} 040$

\begin{tabular}{|l|l|l|l|l|l|l|l|l|l|l|l|l|l|l|l|}
\hline \multicolumn{11}{|c|}{ VME Data bits } \\
\hline 15 & 14 & 13 & 12 & 11 & 10 & 9 & 8 & 7 & 6 & 5 & 4 & 3 & 2 & 1 & 0 \\
\hline 0 & 0 & 0 & 0 & 0 & 0 & 0 & 0 & GIE & 0 & 0 & 0 & 0 & IL2 & IL1 & IL0 \\
\hline
\end{tabular}

Note: IL2 - IL0 is 3-bit VME interrupt level (1...7 - valid, default - 5),

GIE - global interrupt enable (1 - enable, 0 - disable, default - 0)

Table 8 Status ID vector table VT, Base address 0xF800

\begin{tabular}{|c|c|c|}
\hline Input \# & Memory Address & Default Status ID Vector \\
\hline 0 & Base + 0x0010 & 0x08 \\
\hline 1 & Base + 0x0012 & 0x09 \\
\hline
\end{tabular}




\begin{tabular}{|c|c|c|}
\hline 2 & Base + 0x0014 & 0xOA \\
\hline 3 & Base $+0 \times 0016$ & 0xOB \\
\hline 4 & Base + 0x0018 & 0x0C \\
\hline 5 & Base + 0x001A & 0xOD \\
\hline 6 & Base + 0x001C & 0xOE \\
\hline 7 & Base + 0x001E & 0xOF \\
\hline
\end{tabular}

Note: Value of the status ID vector can be anything from 1 to 255,0 - invalid

\section{- Timing module}

The timing module provides four sets of CROC compatible LVDS output signals and has four modes of operation:

- MTM - output clock is synchronous to the MTM clock, other output signals are generated by internal sequencer triggered by TCALB signal from the MTM or by a software command. The sequencer will also be triggered by an external signal at the trigger input (T). SGATE and CNRST can be used as interrupts for the DAQ system. A signal at the trigger input $\mathrm{T}$ is also propagated to the trigger output.

O INT - output signals are generated by internal sequencer synchronously with $53.1047 \mathrm{MHz}$. SGATE, CNRST and TCALB signals are generated periodically at the selected repetition frequency $(0.5 \mathrm{~Hz}$. to $52 \mathrm{kHz})$ or by a software command in the single sequence mode. SGATE and TCALB have programmable settings.

- EXT - SGATE, CNRST and TCALB signals can be provided externally using LVTTL inputs or generated using VME commands.

○ DAQ - special mode which simulates MINERvA front-end and detects front-end trigger. In this mode the CRIM does not generate any output timing signals.

A block diagram of the timing module is shown in Fig. 6. In the MTM mode the PLL circuit is locked to the incoming RF signal from the MINOS timing system. In this mode timing sequencer triggered by the MTM TCALB signal generates internal CNRST, SGATE and TCALB signals. The CNRST and SGATE signals are propagated to the CRIM RJ-45 outputs. The TCALB signal is not propagated, but available as LVTTL signal at the Lemo output marked "C controlled by the bit 15 of the SGATE width register GW (0xC020). When bit 15 is set to one, the TCALB is generated during the sequence. By default this bit is set to zero.

In the DAQ mode encoded timing signal from the DAQ loop is supplied to the PLL reference input. In EXT and INT modes internal clock frequency is provided by a free running quartz oscillator (53.1047 MHz). The external input trigger signal TRIG (labeled T on the front 
panel) is permanently connected to the interrupter Input 0 and can be used in any timing mode. Inputs $1 . . .4$ of the interrupter are connected to the signal multiplexer output, and, therefore, they depend on the timing mode selected. Input 5 is connected to the DAQ loop test module and can be used in a self-triggering DAQ system. The LVTTL output labeled T provides a trigger signal generated by the VME commands (fast trigger or start sequencer) or a copy of the T input signal in the MTM mode. The LVTTL outputs labeled G, R and C correspond to SGATE, CNRST and TCALB signals of the signal multiplexer respectively. The LVTTL inputs labeled T, G, R and C

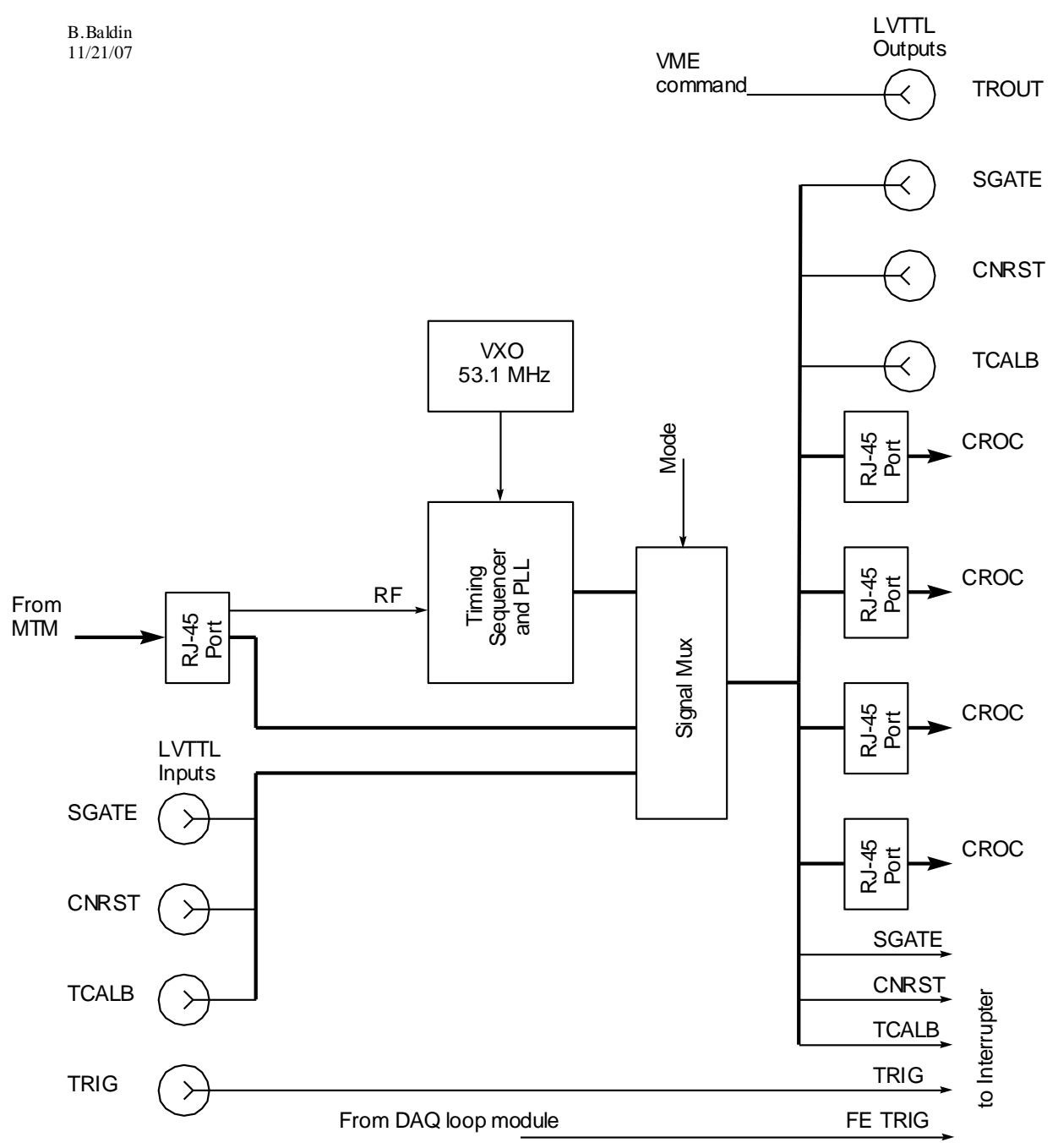

Fig. 6 Timing module

can be used to supply external trigger, SGATE, CNRST and TCAL signals respectively. Note that the input labeled $\mathrm{T}$ has a termination resistor of $1 \mathrm{~K}$ and the other three inputs are terminated with a $50 \mathrm{ohm}$ resistor.

Note that in the EXT timing mode input signals are synchronized to the internal 53.1047 MHz clock. This will cause variation of the delay between LVTTL input and 
corresponding LVTTL output signal. The duration of the output signal will be multiple of $18.9 \mathrm{~ns}$. The following registers provide settings for the timing module:

Timing setup register $\mathrm{TS}, 0 \mathrm{xC} 010$

\begin{tabular}{|l|l|l|l|l|l|l|l|l|l|l|l|l|l|l|l|}
\hline \multicolumn{11}{|c|}{ VME Data bits } \\
\hline 15 & 14 & 13 & 12 & 11 & 10 & 9 & 8 & 7 & 6 & 5 & 4 & 3 & 2 & 1 & 0 \\
\hline M3 & M2 & M1 & M0 & F11 & F10 & F9 & F8 & F7 & F6 & F5 & F4 & F3 & F2 & F1 & F0 \\
\hline
\end{tabular}

Note: F11...F0 frequency select bits (F11- F8 $\sim 0.5 \mathrm{~Hz}$ to $4 \mathrm{~Hz}$ range, F7 - F0 $400 \mathrm{~Hz}$ to $52 \mathrm{kHz}$ range, all zero - single sequence mode); M3...M0 - mode select bits (0x8 - MTM, 0x4 - INT, 0x2- Ext, 0x1 - DAQ)

SGATE width register GW, 0xC020

\begin{tabular}{|l|l|l|l|l|l|l|l|l|l|l|l|l|l|l|l|}
\hline \multicolumn{11}{|c|}{ VME Data bits } \\
\hline 15 & 14 & 13 & 12 & 11 & 10 & 9 & 8 & 7 & 6 & 5 & 4 & 3 & 2 & 1 & 0 \\
\hline TC & $\mathrm{X}$ & $\mathrm{X}$ & $\mathrm{X}$ & $\mathrm{X}$ & $\mathrm{X}$ & $\mathrm{X}$ & $\mathrm{X}$ & $\mathrm{X}$ & $\mathrm{G} 6$ & $\mathrm{G} 5$ & $\mathrm{G} 4$ & G3 & G20 & G1 & G0 \\
\hline
\end{tabular}

Note: G0...G6 - gate width select bits in 150.6 ns steps in INT mode, X - Don't care TC - sequencer control bit ( 1 - enable TCALB, 0 - disable TCALB)

TCALB pulse delay register TP, 0xC030

\begin{tabular}{|l|l|l|l|l|l|l|l|l|l|l|l|l|l|l|l|}
\hline \multicolumn{11}{|c|}{ VME Data bits } \\
\hline 15 & 14 & 13 & 12 & 11 & 10 & 9 & 8 & 7 & 6 & 5 & 4 & 3 & 2 & 1 & 0 \\
\hline $\mathrm{X}$ & $\mathrm{X}$ & $\mathrm{X}$ & $\mathrm{X}$ & $\mathrm{X}$ & $\mathrm{X}$ & $\mathrm{D} 9$ & $\mathrm{D} 8$ & D7 & D6 & D5 & D4 & D3 & D2 & D1 & D0 \\
\hline
\end{tabular}

Note: D9...D0 - TCALB pulse delay select bits in 18.9 ns steps, , X - Don’t care;

The delay counter starts and stops with the gate signal in INT mode

Software trigger register ST, 0xC040

\begin{tabular}{|l|l|l|l|l|l|l|l|l|l|l|l|l|l|l|l|}
\hline \multicolumn{11}{|c|}{ VME Data bits } \\
\hline 15 & 14 & 13 & 12 & 11 & 10 & 9 & 8 & 7 & 6 & 5 & 4 & 3 & 2 & 1 & 0 \\
\hline 0 & 0 & 0 & 0 & 0 & 1 & 0 & 0 & 0 & 0 & 0 & 0 & 0 & 1 & 0 & 0 \\
\hline
\end{tabular}

Note: Writing 0x0404 to this register generates 18.9 ns trigger output pulse

Software TCALB register SP, 0xC050

\begin{tabular}{|l|l|l|l|l|l|l|l|l|l|l|l|l|l|l|l|}
\hline \multicolumn{11}{|c|}{ VME Data bits } \\
\hline 15 & 14 & 13 & 12 & 11 & 10 & 9 & 8 & 7 & 6 & 5 & 4 & 3 & 2 & 1 & 0 \\
\hline 0 & 0 & 0 & 0 & 0 & 1 & 0 & 0 & 0 & 0 & 0 & 0 & 0 & 1 & 0 & 0 \\
\hline
\end{tabular}


Note: Writing 0x0404 to this register generates 18.9 ns TCALB pulse in EXT mode

Software SGATE register GS0, 0xC060

\begin{tabular}{|l|l|l|l|l|l|l|l|l|l|l|l|l|l|l|l|}
\hline \multicolumn{11}{|c|}{ VME Data bits } \\
\hline 15 & 14 & 13 & 12 & 11 & 10 & 9 & 8 & 7 & 6 & 5 & 4 & 3 & 2 & 1 & 0 \\
\hline 0 & 0 & 0 & 0 & 0 & 1 & 0 & 0 & 0 & 0 & 0 & 0 & 0 & 0 & $F$ & $R$ \\
\hline
\end{tabular}

Note: Writing 0x0401 to this register starts SGATE pulse; writing 0x0402 stops SGATE pulse in EXT mode

Software CNRST register CR, 0xC080

\begin{tabular}{|l|l|l|l|l|l|l|l|l|l|l|l|l|l|l|l|}
\hline \multicolumn{11}{|c|}{ VME Data bits } \\
\hline 15 & 14 & 13 & 12 & 11 & 10 & 9 & 8 & 7 & 6 & 5 & 4 & 3 & 2 & 1 & 0 \\
\hline 0 & 0 & 0 & 0 & SS & 0 & CR & 0 & 0 & 0 & 0 & 0 & SS & 0 & CR & 0 \\
\hline
\end{tabular}

Note: Writing 0x0202 to this register generates counter reset pulse in EXT mode;

Writing 0x0808 to this register starts single sequence of CNRST, SGATE and TCALB in

INT or MTM mode when no frequency is selected (F11...F0 are set to zero)

General purpose software register GR, 0xC0A0

\begin{tabular}{|l|l|l|l|l|l|l|l|l|l|l|l|l|l|l|l|}
\hline \multicolumn{11}{|c|}{ VME Data bits } \\
\hline 15 & 14 & 13 & 12 & 11 & 10 & 9 & 8 & 7 & 6 & 5 & 4 & 3 & 2 & 1 & 0 \\
\hline $\mathrm{X}$ & $\mathrm{x}$ & $\mathrm{x}$ & $\mathrm{x}$ & $\mathrm{x}$ & $\mathrm{x}$ & $\mathrm{x}$ & $\mathrm{x}$ & $\mathrm{x}$ & $\mathrm{x}$ & $\mathrm{x}$ & $\mathrm{x}$ & $\mathrm{x}$ & $\mathrm{x}$ & $\mathrm{x}$ & $\mathrm{x}$ \\
\hline
\end{tabular}

Note: This is a general purpose read/write register. Default value $-\mathbf{0 x 0 0 0 0}$

The value of a 28-bit timing counter is latched by the rising edge of the MINOS SGATE signal in two 16-bit registers. This counter is reset approximately every second by the MINOS CNRST signal and provides a timing reference to the MINOS detector timing. The counter is running at the MINERvA MTM clock frequency and its value is valid only in the MTM mode.

Lower 16 bits of the MINOS gate arrival time GT0, 0xC0B0

\begin{tabular}{|l|l|l|l|l|l|l|l|l|l|l|l|l|l|l|l|}
\hline \multicolumn{11}{|c|}{ VME Data bits } \\
\hline 15 & 14 & 13 & 12 & 11 & 10 & 9 & 8 & 7 & 6 & 5 & 4 & 3 & 2 & 1 & 0 \\
\hline T15 & T14 & T13 & T12 & T11 & T10 & T9 & T8 & T7 & T6 & T5 & T4 & T3 & T2 & T1 & T0 \\
\hline
\end{tabular}


Upper 12 bits of the MINOS gate arrival time GT1, 0xC0C0

\begin{tabular}{|c|c|c|c|c|c|c|c|c|c|c|c|c|c|c|c|}
\hline \multicolumn{11}{|c|}{ VME Data bits } \\
\hline 15 & 14 & 13 & 12 & 11 & 10 & 9 & 8 & 7 & 6 & 5 & 4 & 3 & 2 & 1 & 0 \\
\hline 0 & 0 & 0 & 0 & $\mathrm{~T} 27$ & $\mathrm{~T} 26$ & $\mathrm{~T} 25$ & $\mathrm{~T} 24$ & $\mathrm{~T} 23$ & $\mathrm{~T} 22$ & $\mathrm{~T} 21$ & $\mathrm{~T} 20$ & $\mathrm{~T} 19$ & $\mathrm{~T} 18$ & $\mathrm{~T} 17$ & $\mathrm{~T} 16$ \\
\hline
\end{tabular}

Note: T27...T0 bits are latched by the rising edge of the external gate signal

- DAQ loop test module

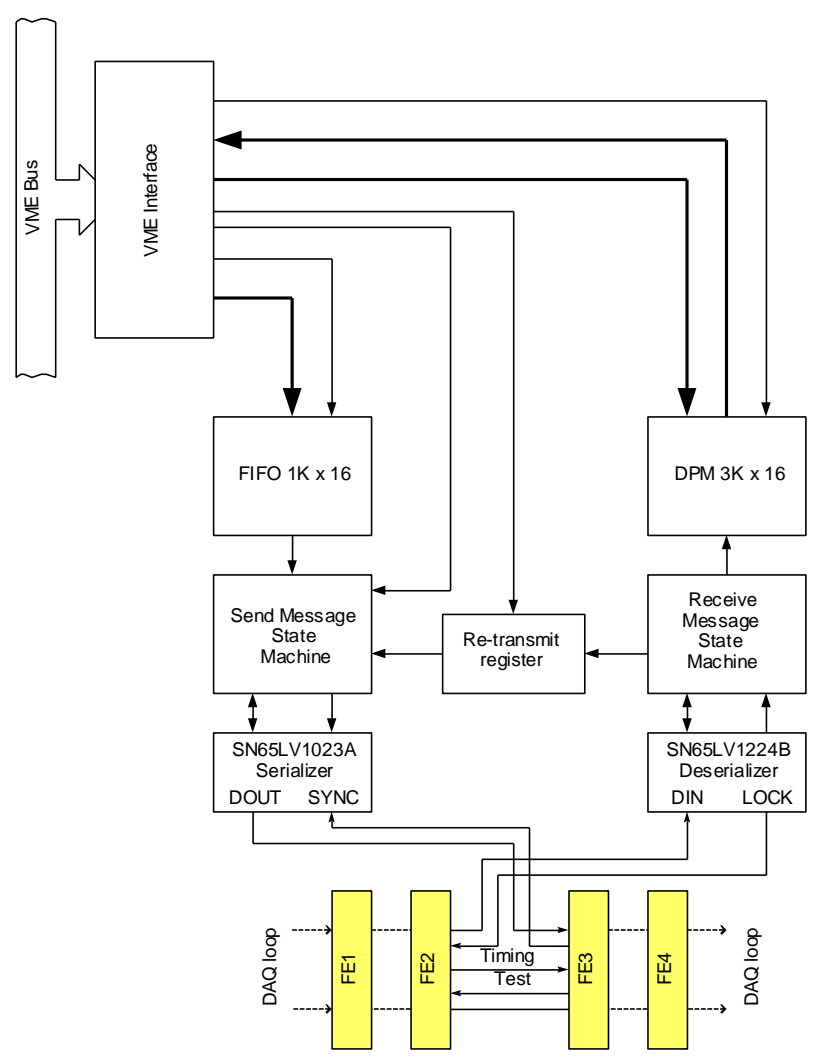

Fig. 7 DAQ loop test module

In the DAQ mode a connection to the external DAQ loop driven by the MINERvA CROC module is required. When inserted in a DAQ loop, this module can be used as a front-end simulator or DAQ loop monitor passing through all messages and storing them in the internal memory. This module also can generate an interrupt when a trigger word is detected in the data stream. A block diagram of the DAQ loop test module is shown in Fig. 7.

The DAQ loop test module has two main modes of operation: pass trough mode and front-end mode. The desired mode is selected by setting to one bit 15 or bit 14 of the DAQ mode control register. In the pass through mode $(\mathrm{TR}=1)$ any message received by the deserializer is stored in the DPM and re-transmitted downstream the DAQ loop via serializer. Usual diagnostics 
of CRC error and DPM overflow is provided. If the front-end trigger is enabled in the pass through mode $(\mathrm{FE}=1)$, the module will generate a signal for the interrupter when a trigger word is received. Each trigger word will be stored in the DPM instead of regular messages. In the front-end mode $(\mathrm{SM}=1)$, any message received by the deserializer is also stored in the DPM. But, after the CRC byte is received, the module generates a response message using the data stored in the FIFO memory. The FIFO is setup in such a way that after the message is sent, the FIFO read pointer is reset. This allows for repeated responses without loading FIFO after each transmission. The DAQ loop test module has the following registers and memory:

Message data Dual Port Memory RD, 0x0000

\begin{tabular}{|c|c|c|c|c|c|c|c|c|c|c|c|c|c|c|c|}
\hline \multicolumn{16}{|c|}{ VME Data bits } \\
\hline 15 & 14 & 13 & 12 & 11 & 10 & 9 & 8 & 7 & 6 & 5 & 4 & 3 & 2 & 1 & 0 \\
\hline \multicolumn{8}{|c|}{$\mathrm{DL}(0)$} & \multicolumn{8}{|c|}{$\mathrm{DL}(1)$} \\
\hline \multicolumn{8}{|c|}{$\operatorname{BYTE}(0)$} & \multicolumn{8}{|c|}{ BYTE(1) } \\
\hline \multicolumn{8}{|c|}{$\ldots$} & \multicolumn{8}{|c|}{$\ldots$} \\
\hline \multicolumn{8}{|c|}{ BYTE(2k-1) } & \multicolumn{8}{|c|}{ BYTE(2k) } \\
\hline
\end{tabular}

Note: DL1, DL0 - data length, BYTE(0) - first byte of the message, BYTE(1) - second byte of the message, BYTE(2k) - last (even) byte of the message.

All messages passing through the DAQ loop test module are stored sequentially in the dual port RAM. The FIFO memory is loaded using a single register.

Trigger data Dual Port Memory RD, 0x0000

\begin{tabular}{|c|c|c|c|c|c|c|c|c|c|c|c|c|c|c|c|}
\hline \multicolumn{16}{|c|}{ VME Data bits } \\
\hline 15 & 14 & 13 & 12 & 11 & 10 & 9 & 8 & 7 & 6 & 5 & 4 & 3 & 2 & 1 & 0 \\
\hline \multicolumn{8}{|c|}{ TRIG(0) } & \multicolumn{8}{|c|}{ TRIG(1) } \\
\hline \multicolumn{8}{|c|}{ TRIG(2) } & \multicolumn{8}{|c|}{ TRIG(3) } \\
\hline \multicolumn{8}{|c|}{$\ldots$} & \multicolumn{8}{|c|}{$\ldots$} \\
\hline \multicolumn{8}{|c|}{$\operatorname{TRIG}(2 \mathrm{k}-1)$} & \multicolumn{8}{|c|}{ TRIG(2k) } \\
\hline
\end{tabular}

All trigger bytes are stored sequentially in the DPM. An interrupt handling routine has to read trigger data after each interrupt and reset DPM address pointer otherwise the DPM overflow flag will eventually be set.

FIFO data register FD, 0x2000

\begin{tabular}{|l|l|l|l|l|l|l|l|l|l|l|l|l|l|l|l|}
\hline \multicolumn{11}{|c|}{ VME Data bits } \\
\hline 15 & 14 & 13 & 12 & 11 & 10 & 9 & 8 & 7 & 6 & 5 & 4 & 3 & 2 & 1 & 0 \\
\hline \multicolumn{11}{|c|}{$\operatorname{BYTE}(0)$} & $\operatorname{BYT}(1)$ \\
\hline
\end{tabular}


Note: BYTE $(0)$ - first byte of the message, BYTE(1) - second byte of the message.

FIFO reset register FR, 0x2008

\begin{tabular}{|l|l|l|l|l|l|l|l|l|l|l|l|l|l|l|l|}
\hline \multicolumn{11}{|c|}{ MME Data bits } \\
\hline 15 & 14 & 13 & 12 & 11 & 10 & 9 & 8 & 7 & 6 & 5 & 4 & 3 & 2 & 1 & 0 \\
\hline 0 & 0 & 0 & 0 & 1 & 0 & 0 & 0 & 0 & 0 & 0 & 0 & 1 & 0 & 0 & 0 \\
\hline
\end{tabular}

Note: Writing 0x0808 to this register resets the FIFO flag

Send message register SM, 0x2010

\begin{tabular}{|l|l|l|l|l|l|l|l|l|l|l|l|l|l|l|l|}
\hline \multicolumn{11}{|c|}{ VME Data bits } \\
\hline 15 & 14 & 13 & 12 & 11 & 10 & 9 & 8 & 7 & 6 & 5 & 4 & 3 & 2 & 1 & 0 \\
\hline 0 & 0 & 0 & 0 & 0 & 0 & 0 & 1 & 0 & 0 & 0 & 0 & 0 & 0 & 0 & 1 \\
\hline
\end{tabular}

Note: Writing 0x0101 to this register sends a message if FIFO is not empty

Status Register SR, 0x2020

\begin{tabular}{|l|l|l|l|l|l|l|l|l|l|l|l|l|l|l|l|}
\hline \multicolumn{11}{|c|}{ VME Data bits } \\
\hline 15 & 14 & 13 & 12 & 11 & 10 & 9 & 8 & 7 & 6 & 5 & 4 & 3 & 2 & 1 & 0 \\
\hline EC & RS & DS & PLO & CM & LS & SY & RF & 0 & DF & FF & EF & 0 & CE & MR & MS \\
\hline
\end{tabular}

Note: MS - Message sent,

MR - Message received,

CE - CRC error,

EF - FIFO not empty flag (0 - empty),

FF $\quad-$ FIFO full flag $(1-$ full $)$,

DF - DPM full flag (1 - full),

$\mathrm{RF} \quad-\mathrm{RF}$ loop monitor (1 - RF present),

SY - Serializer SYNC status (1 - synchronized),

LS - Deserializer LOCK status (1 - locked),

$\mathrm{CM}$ - Common mode level violation

PL0 - CPLD PLL0 status (1 - locked),

DS - Differential control signal received (1 - received),

RS - Reset signal received (1 - received),

EC - Encoded timing command received (1 - received).

Clear status/Reset DPM register CS, 0x2030

\begin{tabular}{|l|l|l|l|l|l|l|l|l|l|l|l|l|l|l|l|}
\hline \multicolumn{11}{|c|}{ VME Data bits } \\
\hline 15 & 14 & 13 & 12 & 11 & 10 & 9 & 8 & 7 & 6 & 5 & 4 & 3 & 2 & 1 & 0 \\
\hline 0 & 0 & 0 & 0 & RP & 0 & CS & 0 & 0 & 0 & 0 & 0 & RP & 0 & CS & 0 \\
\hline
\end{tabular}


Note: $\quad \mathrm{CS}=1$ for Clear Status command, otherwise 0

$\mathrm{RP}=1$ for Reset DPM pointer command, otherwise 0

Send SYNC to serializer register SS, 0x2040

\begin{tabular}{|l|l|l|l|l|l|l|l|l|l|l|l|l|l|l|l|}
\hline \multicolumn{11}{|c|}{ VME Data bits } \\
\hline 15 & 14 & 13 & 12 & 11 & 10 & 9 & 8 & 7 & 6 & 5 & 4 & 3 & 2 & 1 & 0 \\
\hline 0 & 0 & 0 & 0 & 0 & 0 & 0 & 1 & 0 & 0 & 0 & 0 & 0 & 0 & 0 & 1 \\
\hline
\end{tabular}

Note: Writing 0x0101 to this register sends SYNC pulse to the serializer

Read DPM pointer register MP, 0x2050

\begin{tabular}{|l|l|l|l|l|l|l|l|l|l|l|l|l|l|l|l|}
\hline \multicolumn{11}{|c|}{ VME Data bits } \\
\hline 15 & 14 & 13 & 12 & 11 & 10 & 9 & 8 & 7 & 6 & 5 & 4 & 3 & 2 & 1 & 0 \\
\hline P7 & P6 & P5 & P4 & P3 & P2 & P1 & P0 & P15 & P14 & P13 & P12 & P11 & P10 & P9 & P8 \\
\hline
\end{tabular}

Note: P0...P15 - current value of the DPM write pointer (bytes)

Read decoded timing command register DT, 0x2060

\begin{tabular}{|l|l|l|l|l|l|l|l|l|l|l|l|l|l|l|l|}
\hline \multicolumn{11}{|c|}{ VME Data bits } \\
\hline 15 & 14 & 13 & 12 & 11 & 10 & 9 & 8 & 7 & 6 & 5 & 4 & 3 & 2 & 1 & 0 \\
\hline 0 & 0 & 0 & 0 & 0 & 0 & 0 & 0 & C7 & C6 & C5 & C4 & C3 & C2 & C1 & C0 \\
\hline
\end{tabular}

Note: $\quad \mathrm{C} 7 \ldots \mathrm{C} 0$ - bits of the last timing command decoded, start bit not included

DAQ mode control register CR, 0x2070

\begin{tabular}{|l|l|l|l|l|l|l|l|l|l|l|l|l|l|l|l|}
\hline \multicolumn{11}{|c|}{ VME Data bits } \\
\hline 15 & 14 & 13 & 12 & 11 & 10 & 9 & 8 & 7 & 6 & 5 & 4 & 3 & 2 & 1 & 0 \\
\hline TR & SM & CE & FE & 0 & 0 & 0 & 0 & 0 & 0 & 0 & 0 & 0 & 0 & 0 & 0 \\
\hline
\end{tabular}

Note: TR - re-transmit enable (1 - enable)

SM - send message enable (1 - enable)

CE - CRC error enable (1 - enable)

$\mathrm{FE} \quad$ - front-end trigger enable (1 - enable)

\section{VME module implementation}

The current version of the chain readout interface module is implemented as a standard 6Ux160 mm A24D16 VME slave module. It has the following front panel connectors (see Fig. 8): 


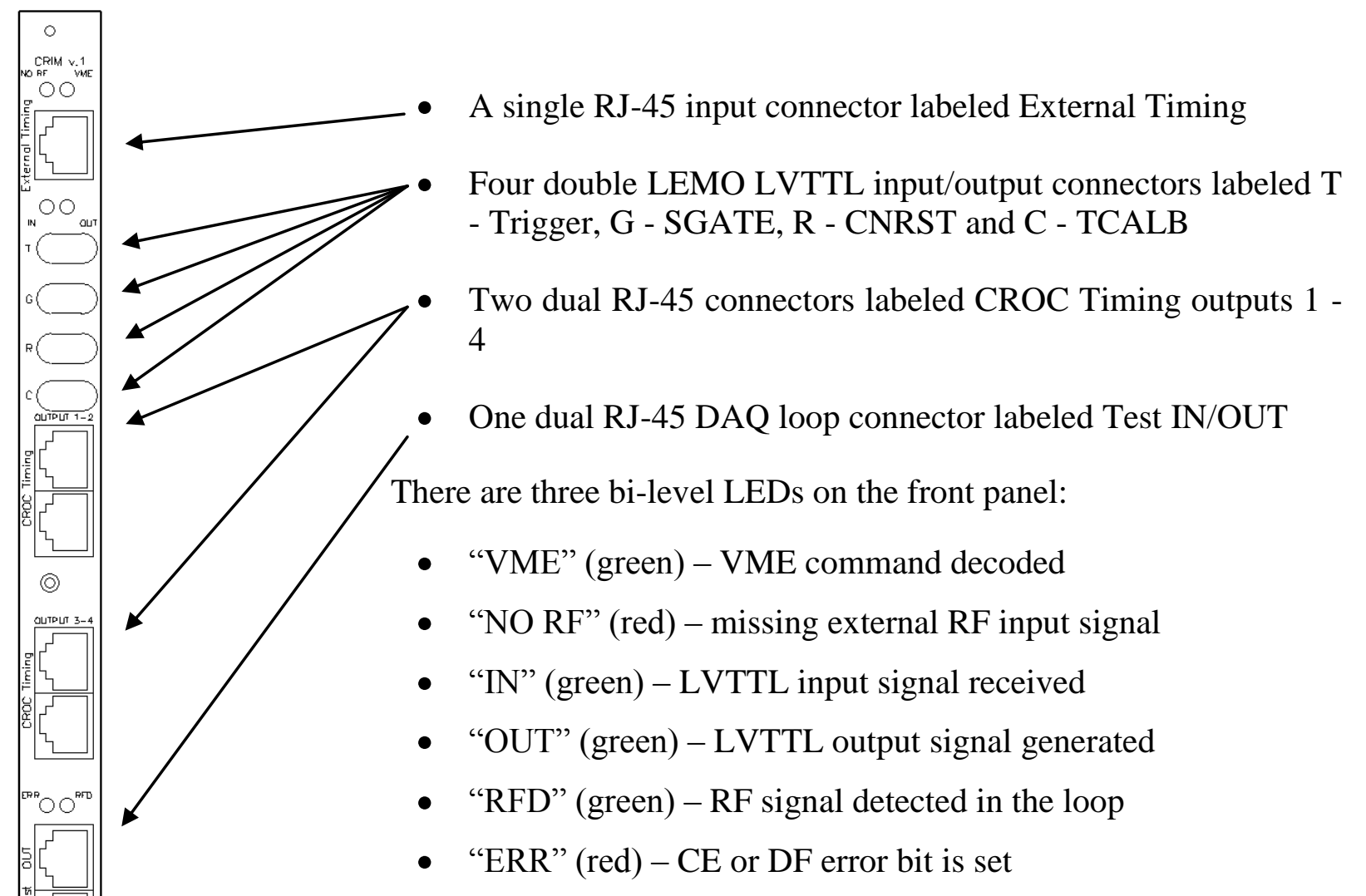

Fig. 8 CRIM front panel

5. Specification

- Power consumption

$-+5 \mathrm{~V} / 0.6 \mathrm{~A}$

- Main clock frequency

- External timing inputs

- CROC timing outputs

- LVTTL inputs impedance

- LVTTL outputs load

- DAQ loop signals

- VME interrupter type

- VME address modifiers

- VME address/data mode

- Test channel FIFO size

- Test channel DPM size

- DAQ loop cable type

- Maximum loop length

- DAQ loop data rate

$-53.1047 \mathrm{MHz}( \pm 200 \mathrm{ppm})$

- LVDS $\left(\right.$ see $^{[4]}$ )

- LVDS $\left(\operatorname{see}^{[4]}\right)$

- $1 \mathrm{~K}, 50 \mathrm{ohm}$

$-1 \mathrm{~K}$

- LVDS/LVTTL

- D08(O)

- 39, 3d, 3e, 3a

- A24D16

- $1 \mathrm{Kx} 16$ bit

- 3Kx16 bit

- CAT5e

$-60 \mathrm{ft}$.

- 13.3 MByte/s 
An eight bit on-board switch selects $64 \mathrm{~K}$ address space for the module. A general VME memory map is shown in Table 9.

Table 9. Chain Readout Interface Module VME map

\begin{tabular}{|c|c|c|c|}
\hline Starting Address & Size & Read/ & Comment \\
\hline Base +000000 & $6 \mathrm{~K}$ & $\mathrm{R}$ & DPM memory, RD \\
\hline Base +002000 & 2 & W & FIFO input data register, FD \\
\hline Base +002008 & 2 & W & FIFO reset register, FR \\
\hline Base +002010 & 2 & W & Send Message register, SM \\
\hline Base +002020 & 2 & $\mathrm{R}$ & Status Register, SR \\
\hline Base +002030 & 2 & w & Clear Status/Reset DPM register, CS \\
\hline Base +002040 & 2 & W & Send sync register, SS \\
\hline Base +002050 & 2 & $\mathrm{R}$ & Read DPM Pointer register, MP \\
\hline Base +002060 & 2 & $\mathrm{R}$ & Read decoded timing command, DT \\
\hline Base +002070 & 2 & $\mathrm{R} / \mathrm{W}$ & Control register, CR \\
\hline Base + 00C010 & 2 & $\mathrm{R} / \mathrm{W}$ & Timing setup register, TS \\
\hline Base + 00C020 & 2 & $\mathrm{R} / \mathrm{W}$ & SGATE width register, GW \\
\hline Base $+00 C 030$ & 2 & $\mathrm{R} / \mathrm{W}$ & TCALB delay register, TP \\
\hline Base $+00 \mathrm{C} 040$ & 2 & W & Software trigger register, ST \\
\hline Base $+00 C 050$ & 2 & W & Software TCALB register, SP \\
\hline Base $+00 C 060$ & 2 & W & Software SGATE start/stop register, GSO \\
\hline Base $+00 \mathrm{C} 080$ & 2 & W & Software CNRST register, CR \\
\hline Base + 00COAO & 2 & $\mathrm{R} / \mathrm{W}$ & General purpose software register, GR \\
\hline Base + 00С0B0 & 2 & $\mathrm{R} / \mathrm{W}$ & Lower 16 bit of MINOS gate timing register, GTO \\
\hline Base + 00CoCo & 2 & $\mathrm{R} / \mathrm{W}$ & Upper 12 bit of MINOS gate timing register, GT1 \\
\hline Base + 00F000 & 2 & $\mathrm{R} / \mathrm{W}$ & Interrupt mask register, IM \\
\hline Base + 00F010 & 2 & $\mathrm{R} / \mathrm{W}$ & Interrupt status register, IS \\
\hline Base + 00F020 & 2 & W & Clear pending interrupts register, $\mathrm{CP}$ \\
\hline Base + 00F040 & 2 & $\mathrm{R} / \mathrm{W}$ & Interrupt configuration register, IC \\
\hline Base + 00F800 & 16 & $\mathrm{R} / \mathrm{W}$ & Status ID vector table memory, VT \\
\hline
\end{tabular}




\section{References}

[1] T.Fitzpatrick, C.Rotolo. "MINOS Master Clock System Preliminary Design Specification," NUMI-NOTR-ELEC-827, Fermilab, November 2000.

[2] B.Baldin, et al. "D0 Muon Readout Electronics Design," IEEE Trans. on Nuclear Science, Vol.44, No.3, pp 363-369, June 1997.

[3] C.Gingu, P.Rubinov. "Minerva Master/Slave Data Acquisition Board Description and Measurement Result," MINERvA Document Database \#1569-v1, http://minervadocdb.fnal.gov,Fermilab, June 2005.

[4] B.Baldin. "MINERvA Chain Readout Controller," MINERvA Document Database \#773-v2, http://minerva-docdb.fnal.gov, Fermilab, May 2006. 